\title{
Numerical Analysis of Roles of Depletion Layer Width in Semiconductor Gas Sensor Using the Gradient-Distributed Oxygen Vacancy Model
}

\author{
Jianqiao LIU ${ }^{1 *}$, Wencong GENG $^{2}$, Guohua JIN $^{1}$, Zhaoxia ZHAI ${ }^{1}$ \\ ${ }^{1}$ College of Information Science and Technology, Dalian Maritime University, Linghai Road 1, Ganjingzi District, Dalian \\ 116026, Liaoning, China P.R. \\ ${ }^{2}$ School of Computer Science and Engineering, University of Electronic Science and Technology of China, Xiyuan Road \\ 2006, Hightech District, Chengdu 611731, Sichuan, China P.R.
}

crossref http://dx.doi.org/10.5755/j01.ms.25.1.19129

Received 27 September 2017; accepted 26 January 2018

\begin{abstract}
The roles of depletion layer width in semiconductor gas sensors are quantitatively discussed based on the model of gradient-distributed oxygen vacancies. Several literatures are employed to provide experimental basis of the relationship between depletion layer width and gas sensing characteristics of semiconductor devices. Four series of gas sensors are illustrated and their depletion layer widths are controlled by three different techniques, such as controlling of the dopant amount, sintering in various procedures and usage of various dopants. The model shows a good applicability in analyzing the roles of the depletion layer width in semiconductor gas sensors, as long as the grain radius is larger than the depletion layer width, especially when it is controlled by the doping amount of the foreign elements.

Keywords: gas sensor, depletion layer, oxygen vacancy, semiconductor.
\end{abstract}

\section{INTRODUCTION}

Semiconductors, such as $\mathrm{SnO}_{2}, \mathrm{ZnO}$ and $\mathrm{WO}_{3}$, are considered to be good choices for core elements in gas sensing devices $[1-3]$. The grains of materials in these sensors are responsible for transducing gaseous environment into electrical energy by the variation of sensor resistance, which is dominated by the depletion layer. This region is responsible for a larger resistivity than the grain bulk, because it contains few free electrons. In this surface layer of the grain, almost all free electrons are captured by the acceptors on the grain surface, leaving a depleted region, where a potential barrier for electrons is established [4]. When exposed to stimulant gases, acceptors are consumed by gas molecules. Consequently, some of captured electrons are released back into the depletion layer, diminishing the resistance of this region [5].

The existence of depletion layer was fundamental in both practical and theoretical investigations because it acts as the sensitive part of the semiconductors. Xu C. proposed a neck-controlled model for porous $\mathrm{SnO}_{2}$ elements. A depletion layer width $(w)$ of $3 \mathrm{~nm}$ was apparently estimated on the basis of the grain size dependences of the sensor resistance and response to reducing gases [6]. However, Malagù's research generated another value of $14.4 \mathrm{~nm}$, by using a Schottky contact model of semiconductor films [7]. Recently, Liu proposed a mathematical method for a more convenient and precise calculation of the depletion layer width based on the dependence of gas-sensing characteristics on the donor additive amount [1]. For $\mathrm{WO}_{3}$ sensors, the response mechanism could be explained by the depletion layer with a variable width [8]. The value has been reported as $12.5 \mathrm{~nm}$ in air and it expanded to 15 and $16.5 \mathrm{~nm}$ when exposed to 5 and $10 \mathrm{ppm} \mathrm{NO}_{2}$ gas [3]. Malagù also found that the width of depletion layer was dependent upon electrical frequency [9]. Likewise, Wang used to observe a width of $15 \mathrm{~nm}$ for the depletion layer of pure $\mathrm{ZnO}$ nanorods [10] and later this value was proved by E. Wongrat's Aucoated $\mathrm{ZnO}$ nanostructured sensors [2]. Although there have been some studies on the depletion layer width of gassensitive semiconductors, a study specific to the roles of the depletion layer width is scarce. Moreover, the existent knowledge is too qualitative. The quantitative analysis is expected.

In the previous works [11-13], the model of gradientdistributed oxygen vacancies (GDOV) was then proposed based on the influence of cooling rate on electrical performances of the semiconductor gas sensitive thin films. The sensor electrical properties were formulated as functions of the grain radius $R_{C}$ and depletion layer width $w$, as expressed Eq 1 and Eq. 2.

$$
\frac{R}{R_{0}}=\exp \frac{q^{2} N_{V S}}{\varepsilon k T m^{2} \cosh \left(m R_{C}\right)}\left\{\begin{array}{c}
\cosh \left(m R_{C}\right) \\
-\cosh \left[m\left(w-R_{C}\right)\right] \\
+m w \sinh \left[m\left(w-R_{C}\right)\right]
\end{array}\right\}
$$

$S=\frac{R_{a}}{R_{g}}=\exp \left[\frac{\alpha q^{2} w^{2} N_{V S}}{\varepsilon k T m R_{C}} \tanh \left(m R_{C}\right)\right]$,

where $R$ and $S$ are the sensor resistance and response to reducing gas, respectively. $R_{0}$ is the flat-band resistance of the central bulk of a $\mathrm{SnO}_{2}$ grain. $q, k, T$ and $e$ are the elementary charge, Boltzmann constant, operating temperature and permittivity, respectively. The parameter $m$ indicates the end temperature of the ideal cooling process. $\alpha$ is a coefficient that indicates the type or concentration of the 
stimulant gases. Thus, the roles of depletion layer width in gas sensing characteristics of semiconductive gas sensors can be analyzed on the basis of the model.

In the present work, the roles of depletion layer width in semiconductor gas sensors are discussed based on the model of the gradient-distributed oxygen vacancy. Several semiconductor gas sensors are selected from the previous literatures, where the depletion layer width of the sensors was controlled in different techniques. The dependence of gas sensing characteristics on the depletion layer width is quantitatively described by the GDOV model. The simulated sensor resistance and response show good agreement with the experimental results.

\section{PREPARATION ROUTES OF SENSOR SAMPLES}

It is known that there are several ways to adjust the depletion layer width of semiconductor gas sensor. Incorporation and sintering control are the most popular techniques. Four types of gas sensors are selected for the present discussion in the role of the depletion layer width, as listed in Table 1.

Table 1. List of selected gas sensors and their preparation routes

\begin{tabular}{|c|c|c|c|}
\hline Series & Sensor type & $\begin{array}{c}\text { Method to change } \\
\text { depletion layer } \\
\text { width }\end{array}$ & Reference \\
\hline$\# 1$ & $\mathrm{SnO}_{2}$ thin film & $\begin{array}{c}\text { Incorporation of } \\
\text { Sb addition }\end{array}$ & {$[1]$} \\
\hline$\# 2$ & $\begin{array}{c}\mathrm{ZnO} \\
\text { nanostructured } \\
\text { sensor }\end{array}$ & $\begin{array}{c}\text { Incorporation of } \\
\text { Au nanoparticles }\end{array}$ & {$[2]$} \\
\hline$\# 3$ & $\mathrm{WO}_{3}$ pellet & $\begin{array}{c}\text { Controlled } \\
\text { sintering }\end{array}$ & {$[3]$} \\
\hline$\# 4$ & $\mathrm{SnO}_{2}$ pellet & $\begin{array}{c}\text { Incorporation of } \\
\text { various elements }\end{array}$ & {$[6]$} \\
\hline
\end{tabular}

Series \#1 from Liu [1] was $\mathrm{SnO}_{2}$ thin films spin-coated on alumina substrates via a sol-gel route. The thin films were sintered at $600{ }^{\circ} \mathrm{C}$ for $2 \mathrm{~h}$. Sb addition was incorporated into $\mathrm{SnO}_{2}$ thin films to obtain various depletion layer widths from 4.2 to $2.3 \mathrm{~nm}$, which were calculated based on the dependence of thin film resistance on the $\mathrm{Sb}$ doping amount.

Series \#2 was prepared by E Wongrat [2], who incorporated $\mathrm{Au}$ nanoparticles into $\mathrm{ZnO}$ nanostructured gas sensor, which was prepared by thermal oxidation technique on alumina substrates. The sensor was sintered at the temperature of $500{ }^{\circ} \mathrm{C}$ for $24 \mathrm{~h}$ to form a $\mathrm{ZnO}$ nanostructure. Adding Au nanoparticles on the surface of $\mathrm{ZnO}$ grains was performed by sputtering technique to change the depletion layer width.

Series \#3 was a conventional sintered block type sensor fabricated by J. Tamaki [3] for detection of $\mathrm{NO}$ and $\mathrm{NO}_{2}$ gases. $\mathrm{WO}_{3}$ powder was prepared from aqueous solution before it was pressed into a sensing pellet and sintered at $300-550{ }^{\circ} \mathrm{C}$ for $3 \mathrm{~h}$. Different sintering processes were applied to the sensors to adjust the depletion layer width.

Series \#4 was from $\mathrm{C} \mathrm{Xu}$, who prepared porous sensor elements by pasting $\mathrm{SnO}_{2}$ powder on an alumina tube sintered at $700{ }^{\circ} \mathrm{C}$ for $4 \mathrm{~h}$. Foreign oxides were added by an impregnation method to control the grain size of the
$\mathrm{SnO}_{2}$ sensor, in which the grain size effects of sensor resistance and response were observed. A depletion layer width of $3 \mathrm{~nm}$ was concluded for $\mathrm{SnO}_{2}$ grains and this value was considered to be a constant. Therefore, the depletion layer width appears different performances in the $\mathrm{SnO}_{2}$ sensors with various grain sizes.

\section{RESULTS AND DISCUSSION}

The influence of the depletion layer width on gas sensing characteristics of Sb-doped $\mathrm{SnO}_{2}$ thin films (Series \#1) is shown in Fig. 1. The plots are fitted by setting the parameters in Eq. 1 and Eq. 2 as: $N_{V S}=5 \times 10^{25} \mathrm{~m}^{-3}$, $m=0.2 \mathrm{~nm}^{-1}, \quad \varepsilon=10^{-10} \mathrm{~F} / \mathrm{m}, \quad T=573 \mathrm{~K}, \quad \alpha=0.7 \quad$ and $R_{0}=1.25 \Omega$. It is found that the simulations are in good agreement with the experimental observation. The GDOV model shows good potentials in simulating the roles of depletion layer width on the electrical properties of semiconductor gas sensors.

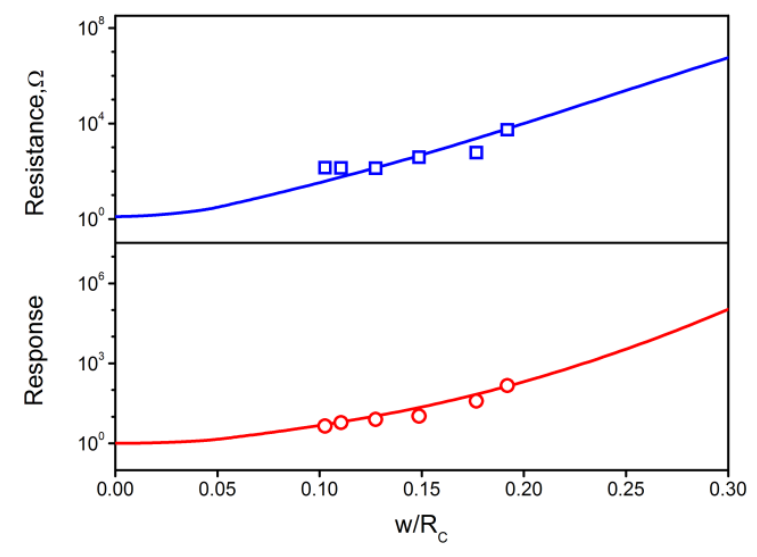

Fig. 1. Dependence of Sb-doped $\mathrm{SnO}_{2}$ thin film characteristics on the depletion layer width at $573 \mathrm{~K}$ [1] and their fitting curves

The sensing characteristics of the $\mathrm{ZnO}$ ethanol sensor (Series \#2) at $573 \mathrm{~K}$ are redrawn in Fig. 2. They are fitted by setting the variables of Eq. 1 and Eq. 2 as: $N_{V S}=10^{25} \mathrm{~m}^{-3}, \varepsilon=8.58 \times 10^{-11} \mathrm{~F} / \mathrm{m}, T=573 \mathrm{~K} . m$ is set to be $0.18 \mathrm{~nm}^{-1}$ and the $\mathrm{C}_{2} \mathrm{H}_{5} \mathrm{OH}$ gas concentration of 500 and $1000 \mathrm{ppm}$ are denoted by $\alpha=0.16$ and $\alpha=0.17$, respectively. It is observed that the simulation fits the experimental resistance and response perfectly. Only one value of $m$ is used since the depletion layer width is solely controlled by the amount of Au impregnation.

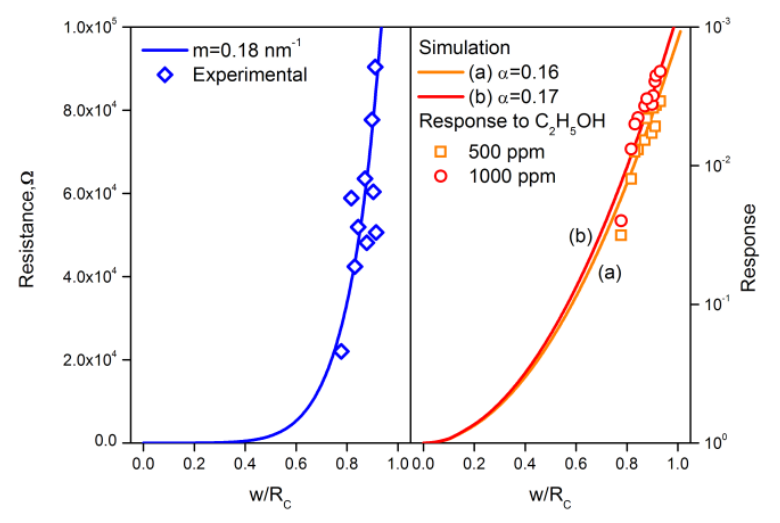

Fig. 2. The resistance and response of $\mathrm{ZnO}$ nanostructure sensor at $573 \mathrm{~K}[2]$ and their simulations 
The $\mathrm{WO}_{3}$ sensor resistance and response of Series \#3 are fitted in Fig. 3 and Fig. 4 by setting the parameters as: $N_{V S}=10^{25} \mathrm{~m}^{-3}, \quad \varepsilon=8.85 \times 10^{-11} \mathrm{~F} / \mathrm{m}, \quad T=573 \mathrm{~K}$ and $R_{0}=31250 \Omega$. Without any interference from the incorporated elements, the pure $\mathrm{WO}_{3}$ resistance is well fitted by the simulations when $m=0.25$ and $0.30 \mathrm{~nm}^{-1}$, which denote their sintering processes. Also, good simulations are observed by fitting the sensor response to 5 and $10 \mathrm{ppm} \mathrm{NO}_{2}$ gas at $573 \mathrm{~K}$ when $\alpha=0.16$ and 0.40 , respectively, as shown in Fig. 4.

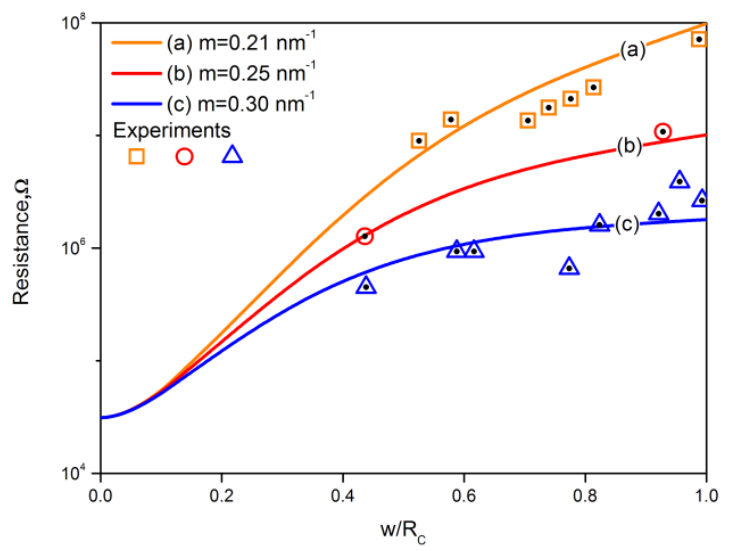

Fig. 3. Resistance of $\mathrm{WO}_{3}$ sensors at $573 \mathrm{~K}$ [3] and the calculated fitting curves

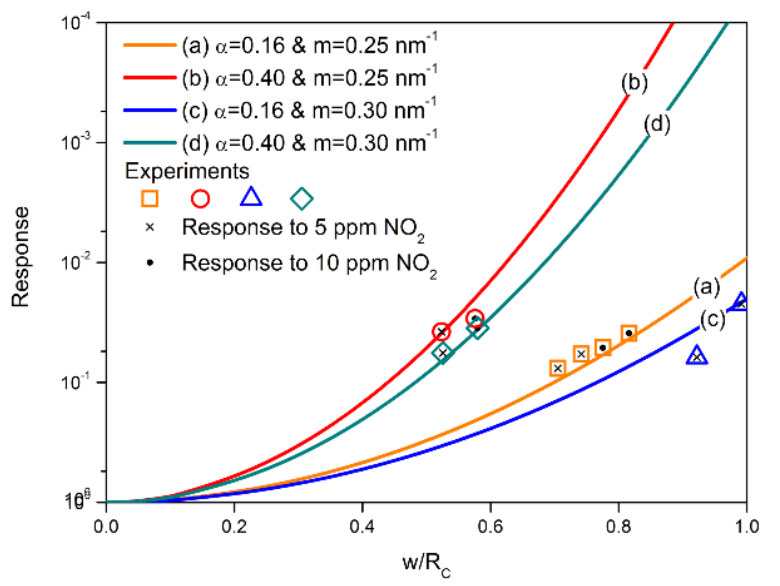

Fig. 4. Response of $\mathrm{WO}_{3}$ sensors to $\mathrm{NO}_{2}$ gas at $573 \mathrm{~K}$ [3] the calculated fitting curves

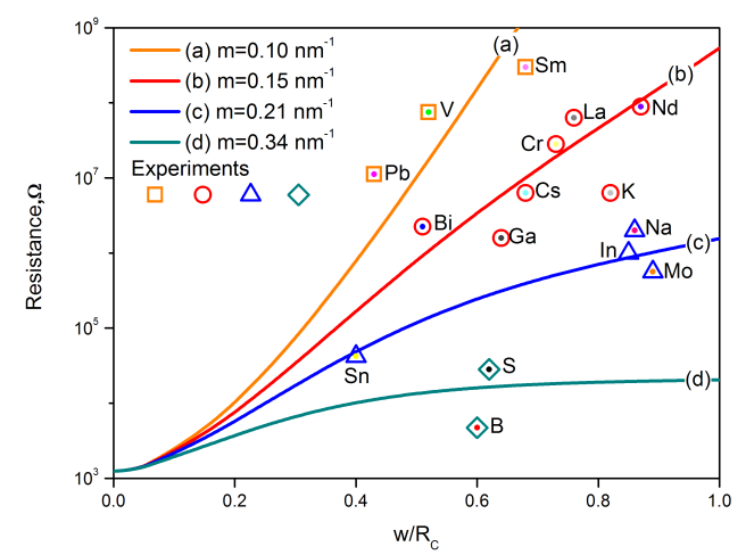

Fig. 5. Simulated sensor resistance against $w / R_{C}$ and its fitting with experimental resistance of $\mathrm{SnO}_{2}$ elements at $573 \mathrm{~K}$ [6]
For the gas sensors of Series \#4, various elements were incorporated when the $\mathrm{SnO}_{2}$ gas sensors were prepared. The results are redrawn in Fig. 5 in the coordinates of resistance against $w / R_{C}$. The simulated curves with $m=0.10,0.15$, 0.21 and $0.34 \mathrm{~nm}^{-1}$ are plotted to fit the resistance at $573 \mathrm{~K}$. $R_{0}$ is set to be $1250 \Omega$. Most plots locate themselves around the simulations, though they are separated into several groups. It is known that the resistance of gas sensors is not only controlled by the grain size, but also influenced by the foreign species. These additives can significantly change the sensor performance by surface modification, acting as catalyst for a chemical reaction or controlling the grain size [14]. It is very difficult to identify the contribution of each dopant, which is not taken into consideration in the model. However, if some of the foreign elements have the similar effect on the sensor resistance, they can be treated as one group. In this case, the simulation presents its applicability provided that the incorporated elements have similar effects on the gas sensing properties.

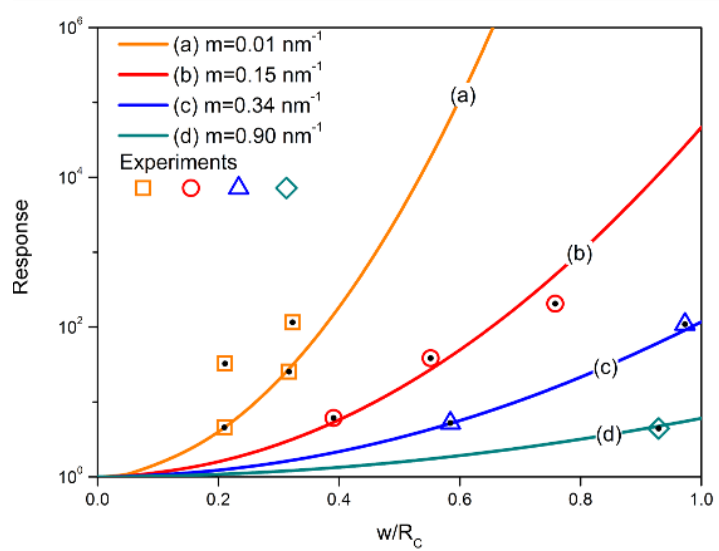

Fig. 6. Simulated sensor response against $w / R_{C}$ and its fitting with actual response of $\mathrm{SnO}_{2}$ element to $800 \mathrm{ppm} \mathrm{H}_{2}$ gas at $573 \mathrm{~K}[6]$

On the other hand, Fig. 6 shows the fitting of the sensor response to $800 \mathrm{ppm} \mathrm{H}_{2}$ of the pure $\mathrm{SnO}_{2}$ elements. They were fabricated in various sintering conditions, which could be denoted by $m$ in the present model. Therefore, several values of $m$ are used to simulate the relationship between sensor response and $w / R_{C}$, as shown in Fig. 6 . The parameters are set to be: $T=573 \mathrm{~K}$ and $\alpha=0.25$. The simulations appear good fitting with the experimental response. However, the shape of the simulated curve is found almost the same as Line (a) if $m$ is less than $0.01 \mathrm{~nm}$ [1]. Hence, there is little possibility for the present model to cover all the actual data of response.

The roles of depletion layer width in semiconductor gas sensors were analyzed by the GDOV model. Generally, there are three methods used to adjust the width of depletion layer, namely: (a) controlling the amount of incorporated elements, (b) sintering in various procedures and (c) usage of various dopants. Then, the calculated gas sensing properties are validated by several typical literatures, in which various types of materials and fabrication procedures were used. For the case of Sb-doped $\mathrm{SnO}_{2}$ thin film and $\mathrm{ZnO}$ sensors (Series \#1 and \#2), all the samples were sintered in the same process while the doping amount of the exclusive foreign element was controlled. The present model shows a perfect fitting in these events. In the next situation of Series 
\#3, various sintering processes were employed to prepare gas sensors of the $\mathrm{WO}_{3}$ blocks. The model is also validated by changing $m$ values, which originally indicate the end temperature of the annealing processes. Therefore, the good fittings are also observed in Fig. 3 and Fig. 4. For the last case, it has to be reserved that the effects of various dopants on gas sensor performances are not taken into consideration in this model. Hence, the fitting shows to be less precise to C. Xu's sensors, which incorporated more than ten types of foreign elements. However, the model is still applicable if the effects of additives on sensor properties are grouped. It is noted that the current calculation is valid as long as the grain radius is larger than the depletion layer width. In other situations, the Eq. 1 and Eq. 2 are degraded and they have been discussed in another work[11].

\section{CONCLUSIONS}

The roles of the depletion layer width in semiconductor gas sensors are analyzed based on the model of GDOV. Some sensor performances of $\mathrm{SnO}_{2}, \mathrm{WO}_{3}$ and $\mathrm{ZnO}$ devices are employed for discussion. The parameter $m$ can be used to indicate the various sintering procedures in the fabrication of gas detectors. A better fitting is observed when the depletion layer width is controlled by the dopant amount than by the sintering technique. It is also suitable for the model when various foreign species are introduced to modify the depletion layer width of semiconductive gas sensors, provided that the effects of incorporated elements are grouped.

\section{Acknowledgments}

This work is financially supported by the National Natural Science Foundation of China (Grant No. 11704055) and the Fundamental Research Funds for the Central Universities (Grant No. 3132016320, 3132016347and 3132017079).

\section{REFERENCES}

1. Liu, J., Liu, X., Zhai, Z., Jin, G., Jiang, Q., Zhao, Y., Luo, C., Quan, L. Evaluation of Depletion Layer Width and Gas-sensing Properties of Antimony-doped Tin Oxide Thin Film Sensors Sensors and Actuators B: Chemical 220 (15) 2015: pp. $1354-1360$. https://doi.org/10.1016/j.snb.2015.07.065

2. Wongrat, E., Hongsith, N., Wongratanaphisan, D., Gardchareon, A., Choopun, S. Control of Depletion Layer Width via Amount of AuNPs for Sensor Response Enhancement in $\mathrm{ZnO}$ Nanostructure Sensor Sensors and Actuators B: Chemical 171 2012: pp. 230-237. https://doi.org/10.1016/j.snb.2012.03.050

3. Tamaki, J., Zhang, Z., Fujimori, K., Akiyama, M., Harada, T., Miura, N., Yamazoe, N. Grain-size Effects in Tungsten Oxide-based Sensor for Nitrogen Oxides Journal of the Electrochemical Society $141(8)$ 1994: pp. $2207-2210$.

\section{https://doi.org/10.1149/1.2055088}

4. Yamazoe, N., Fuchigami, J., Kishikawa, M., Seiyama, T. Interactions of Tin Oxide Surface with $\mathrm{O}_{2}, \mathrm{H}_{2} \mathrm{O}$ and $\mathrm{H}_{2}$ Surface Science 86 (1) 1979: pp. 335-344. https://doi.org/10.1016/0039-6028(79)90411-4

5. Morrison, S.R. Mechanism of Semiconductor Gas Sensor Operation Sensors and Actuators 11(3) 1987: pp. $283-287$. https://doi.org/10.1016/0250-6874(87)80007-0

6. Xu, C., Tamaki, J., Miura, N., Yamazoe, N. Grain Size Effects on Gas Sensitivity of Porous $\mathrm{SnO}_{2}$-based Elements Sensors and Actuators B: Chemical 3(2) 1991: pp. $147-155$. https://doi.org/10.1016/0925-4005(91)80207-Z

7. Malagù, C., Guidi, V., Stefancich, M., Carotta, M.C., Martinelli, G. Model for Schottky Barrier and Surface States in Nanostructured N-type Semiconductors Journal of Applied Physics 91 (2) 2002: pp. 808-814. https://doi.org/10.1063/1.1425434

8. Shi, J., Hu, G., Sun, Y., Geng, M., Wu, J., Liu, Y., Ge, M., Tao, J., Cao, M., Dai, N. WO 3 Nanocrystals: Synthesis and Application in Highly Sensitive Detection of Acetone Sensors and Actuators B: Chemical $156(2)$ 2011: pp. $820-824$ https://doi.org/10.1016/j.snb.2011.02.047

9. Malagù, C., Carotta, M., Gherardi, S., Guidi, V., Vendemiati, B., Martinelli, G. AC Measurements and Modeling of $\mathrm{WO}_{3}$ Thick Film Gas Sensors Sensors and Actuators B: Chemical 108 (1) 2005: pp. 70-74. https://doi.org/10.1016/j.snb.2004.10.049

10. Li, C., Du, Z., Li, L., Yu, H., Wan, Q., Wang, T. SurfaceDepletion Controlled Gas Sensing of ZnO Nanorods Grown at Room Temperature Applied Physics Letters 91 (3) 2007: pp. 032101-032101-3. https://doi.org/10.1063/1.2752541

11. Liu, J., Gong, S., Quan, L., Deng, Z., Liu, H., Zhou, D. Influences of Cooling Rate on Gas Sensitive Tin Oxide Thin Films and a Model of Gradient Distributed Oxygen Vacancies in $\mathrm{SnO}_{2}$ Crystallites Sensors and Actuators B: Chemical 145 (2) 2010: pp. $657-666$. https://doi.org/10.1016/j.snb.2010.01.015

12. Liu, J., Gong, S., Fu, Q., Wang, Y., Quan, L., Deng, Z., Chen, B., Zhou, D. Time-dependent Oxygen Vacancy Distribution and Gas Sensing Characteristics of Tin Oxide Gas Sensitive Thin Films Sensors and Actuators B: Chemical 150 (1) 2010: pp. 330-338. https://doi.org/10.1016/j.snb.2010.06.065

13. Liu, J., Gao, Y., Wu, X., Jin, G., Zhai, Z., Liu, H. Inhomogeneous Oxygen Vacancy Distribution in Semiconductor Gas Sensors: Formation, Migration and Determination on Gas Sensing Characteristics Sensors 17 2017: pp. 1852. https://dx.doi.org/10.3390/s17081852

14. Tamaki, J. High Sensitivity Semiconductor Gas Sensors Sensor Letters 3 (2) 2005: pp. 89-98. https://doi.org/10.1166/s1.2005.023 